\title{
Using Ethereum blockchain to store and query pharmacogenomics data via smart contracts
}

\author{
Gamze Gürsoy ${ }^{1 \dagger}$, Charlotte M. Brannon ${ }^{2 \dagger}$ and Mark Gerstein $1,2,3^{*}$
}

\begin{abstract}
Background: As pharmacogenomics data becomes increasingly integral to clinical treatment decisions, appropriate data storage and sharing protocols need to be adopted. One promising option for secure, high-integrity storage and sharing is Ethereum smart contracts. Ethereum is a blockchain platform, and smart contracts are immutable pieces of code running on virtual machines in this platform that can be invoked by a user or another contract (in the blockchain network). The 2019 iDASH (Integrating Data for Analysis, Anonymization, and Sharing) competition for Secure Genome Analysis challenged participants to develop time- and space-efficient Ethereum smart contracts for gene-drug relationship data.
\end{abstract}

Methods: Here we design a specific smart contract to store and query gene-drug interactions in Ethereum using an index-based, multi-mapping approach. Our contract stores each pharmacogenomics observation, a gene-variant-drug triplet with outcome, in a mapping searchable by a unique identifier, allowing for time and space efficient storage and query. This solution ranked in the top three at the 2019 IDASH competition. We further improve our "challenge solution" and develop an alternate "fastQuery" smart contract, which combines together identical gene-variant-drug combinations into a single storage entry, leading to significantly better scalability and query efficiency.

Results: On a private, proof-of-authority network, both our challenge and fastQuery solutions exhibit approximately linear memory and time usage for inserting into and querying small databases ( $<1,000$ entries). For larger databases (1000 to 10,000 entries), fastQuery maintains this scaling. Furthermore, both solutions can query by a single field ("0-AND") or a combination of fields ("1- or 2-AND"). Specifically, the challenge solution can complete a 2-AND query from a small database (100 entries) in $35 \mathrm{~ms}$ using $0.1 \mathrm{MB}$ of memory. For the same query, fastQuery has a 2-fold improvement in time and a 10 -fold improvement in memory.

Conclusion: We show that pharmacogenomics data can be stored and queried efficiently using Ethereum blockchain. Our solutions could potentially be used to store a range of clinical data and extended to other fields requiring high-integrity data storage and efficient access.

Keywords: Blockchain, Ethereum, Pharmacogenomics data, Smart contract, Secure storage

\footnotetext{
*Correspondence: mark@gersteinlab.org

${ }^{\dagger}$ Gamze Gürsoy and Charlotte M. Brannon contributed equally to this work.

${ }^{1}$ Program in Computational Biology and Bioinformatics, Yale University,

Whitney Avenue, 06520 New Haven, CT, USA

2Department of Molecular Biophysics and Biochemistry, Yale University,

Whitney Avenue, 06520 New Haven, CT, USA

Full list of author information is available at the end of the article
}

(c) The Author(s). 2020 Open Access This article is licensed under a Creative Commons Attribution 4.0 International License, which permits use, sharing, adaptation, distribution and reproduction in any medium or format, as long as you give appropriate credit to the original author(s) and the source, provide a link to the Creative Commons licence, and indicate if changes were made. The images or other third party material in this article are included in the article's Creative Commons licence, unless indicated otherwise in a credit line to the material. If material is not included in the article's Creative Commons licence and your intended use is not permitted by statutory regulation or exceeds the permitted use, you will need to obtain permission directly from the copyright holder. To view a copy of this licence, visit http://creativecommons.org/licenses/by/4.0/. The Creative Commons Public Domain Dedication waiver (http://creativecommons.org/publicdomain/zero/1.0/) applies to the data made available in this article, unless otherwise stated in a credit line to the data. 


\section{Background}

Pharmacogenomics data, which describes the results of particular gene-drug interactions, allows researchers and physicians to predict how specific patients will respond to a given drug based on the genetic variants they possess. For example, a patient may be more prone to toxic effects from a drug because they possess a variant which limits their ability to clear the drug, and therefore should be prescribed an alternative. The Mayo Clinic likens inclusion of pharmacogenomics data in a patient's chart to a "flashing, genomic medical alert band," implying that this data may become as commonplace in basic medical care as wristbands indicating a patient's allergies or fall risk [1]. Given the growing reliance on this data for medical treatment, its corruption or loss, whether intentional or accidental, has the potential to directly impact medical treatments. It is thus of the essence to develop a robust method for storing, sharing, and updating pharmacogenomics databases in a secure, high-integrity fashion.

The importance of securely storing personal genomic data to protect personal privacy has been widely noted [2]. Pharmacogenomics data presents similar concerns for privacy and immutability. For both genomics and pharmacogenomics, data integrity is critical, as loss, corruption, or alteration of the data would have problematic effects (in the case of genomics, making the wrong diagnosis, and in pharmacogenomics, prescribing the wrong drug). Thus, any method for storing and sharing pharmacogenomics data must prevent it from being lost, changed, or corrupted. A balance between accessibility and privacy is also key to both kinds of data. Researchers and physicians must be able to access genomic data without leaking private information about their patients. They also must be able to store and share gene-drug interaction data during clinical trial phases, while respecting proprietary protocol (i.e. within a pharmaceutical company) or simply sharing only with other groups contributing to the study (i.e. within or between academic institutions). These requirements invite development of creative solutions in order to guarantee secure, robust pharmacogenomics databases.

Blockchain technology is growing in popularity to solve secure data storage problems because of its decentralization, distributed architecture, and immutability. Decentralization prevents any single user from controlling the data; distributed architecture eliminates the single point of failure; and immutability prevents alteration of past records. Given the requirements for storing pharmacogenomics data discussed above, blockchain technology is an ideal implementation. Some argue that blockchain is a technology fad, and is being used to solve problems that other, simpler technologies could solve, namely a distributed database [3]. However, according to Kuo et al. ["Blockchain distributed ledger technologies for biomedical and healthcare applications," JAMIA, 2017], blockchain offers features which distributed databases do not, including decentralized management, an immutable audit trail, data provenance, robustness and availability, and security and privacy. These key benefits make blockchain better suited for biomedical applications than other distributed database management systems [4].

A blockchain is a decentralized, distributed, digital ledger comparable to an append-only list linked by cryptographic hashes [5]. The ledger is shared in a "peer-to-peer" network; each node in the network keeps a copy of the list on their computer which syncs to the rest of the network. Nodes in the network submit transactions, which may be verified and added to the chain in a new block. Each block possesses a hash of its contents and the previous block's hash. Thus, one cannot alter the record, as even the smallest alteration will drastically change the downstream block hashes. The blockchain data structure was first introduced in 2008 by Satoshi Nakamoto (pseudonym) as a digital ledger for transactions of Bitcoin, a now infamous cryptocurrency [5]. However, since this initial application blockchain technology has evolved into a more versatile and dynamic technology, now used for tasks ranging from food distribution tracking to music streaming $[6,7]$.

Blockchain is increasingly applied to solve real-world problems because of the transparency, immutability, security, privacy, and disintermediation it provides. To achieve transparency, every transaction on the chain is broadcast to all other users in the network, who mine and verify the transactions. Immutability arises because each block contains the hash of all contents in the previous block, preventing any changes to past transactions. Security is achieved through distributed architecture; the transaction history and data stored on the chain is distributed across many nodes in the network, so there is no single point of failure. User privacy is preserved, in a public blockchain network at least, because users can be anonymous, identifiable only by their wallet address. This technology also eliminates the need for a middle man, such as a bank or a music streaming platform, and allows users to transact directly with other users because there is no need for trust in the network [5].

Many of today's blockchain applications were built to run in Ethereum. Ethereum is a transaction-based state machine which logs modifications to its state in a blockchain. This machine permits development of applications designed for both public and private blockchains through 'smart contracts' [8]. Smart contracts are selfexecutable pieces of code which live in the Ethereum state and trigger transactions when called by a user or another smart contract [9]. Whereas transactions in other blockchain environments, such as bitcoin, were limited in their complexity due to the network state configuration and programming language used, smart contracts are programmed in turing-complete languages such as 
Solidity, an object-oriented language influenced by JavaScript, $\mathrm{C}++$, Python, and PowerShell [10]. Smart contracts offer transparency (users can verify who deployed the program and ensure they are using the correct version of the program) and immutability (the program cannot be altered and any new versions of the program are accessible to all users). A common example of a smart contract is one which allows citizens to vote securely in an election [11]. Yet, there is significant potential to use smart contracts to perform transactions in a variety of contexts and industries.

For the 2019 iDASH competition, we aimed to develop an Ethereum smart contract for storing and querying pharmacogenomics data with time and memory efficiency. While blockchain technology offers several useful features, it is notoriously inefficient and slow when it comes to storing and querying data [5]. Thus, it is challenging to use for everyday applications. Additionally, development and deployment of smart contracts requires command of the Solidity programming language, which has many eccentricities, and working with the continuously evolving Ethereum API. However, we addressed these design and logistical challenges, and presented an index-based, multi-mapping data structure in a Solidity smart contract to store the data. Specifically, we store each pharmacogenomics observation in a mapping searchable by a unique integer identifier. We then keep three additional mappings which store the unique identifiers by gene name, variant number, and drug name, respectively. This design allowed for time and memory efficient data insertion and querying by one to three fields. For the sake of scalability, we developed an alternate solution referred to as fastQuery. In this solution, we store the data for each unique gene-variant-drug combination as a single entry in storage. This eliminated the need to pool data from multiple locations in storage during query. This fastQuery solution exhibited significantly increased time efficiency for querying by one to three fields.

\section{Methods}

In this study we designed a time/space efficient data structure and algorithms to store and query pharmacogenomics within a smart contract in a private Ethereum blockchain. The data was stored on a small blockchain network with only four nodes. Each data point was inserted to our smart contract as a single transaction. All data had to be stored on-chain. No off-chain data storage was allowed.

\section{Blockchain technology explained}

Here we provide a primer on blockchain technology for those unfamiliar with the basics. For a more rigorous treatment of this material, the reader should consult the bitcoin white paper and the ethereum yellow paper $[5,8]$.
As outlined earlier, a blockchain is a secure data structure shared in a peer-to-peer network [Fig. 1a-b]. The chain is made up of blocks of data forward-linked by hashes. Each block in the chain contains a header and a list of valid transactions. The header includes several fields relevant to both the integrity of the data structure (e.g. the timestamp), and the parameters of the network (e.g. mining parameters). These fields include the block timestamp, block number, mining parameters, and the hash of the previous block's contents (which links one block to the next). Users or nodes can submit transactions which modify the state of the network, for example by sending value from one user address to another, or storing a value within a smart contract (discussed further below).

The network consensus mechanism determines which user in the network will append the transactions to the chain as a new block. This is a critical process; if it were to fail, the validity of the added block would be compromised. The most prominent consensus mechanisms include proof of work, proof of stake, and proof of authority. In a proof of work (PoW) network, nodes "mine" blocks; that is, they exert computational energy to identify values which, when added to the incoming block's contents, yields a block hash below that of a set network parameter referred to as the difficulty. The difficulty is a network-wide parameter which can be varied in order to regulate the rate of block formation. The proof of work mechanism is critical to public cryptocurrency blockchain networks such as Bitcoin because it sets a cost for modifying the blockchain, thereby deterring bad actors from corrupting the chain [5]. Many have criticized the PoW mechanism for being unsustainable in the long run because of the large amounts of energy required to perform computational mining [12]. To address this criticism, the proof of stake mechanism has been proposed [13]. With proof of stake, a network algorithm determines which node will add the block to the chain based on the node's stake, a combination of parameters including their account balance [14]. The PoS mechanism does not require heavy computation, thereby reducing the energy usage in the network. These consensus mechanisms are essential for a public blockchain network where anyone in the world can run a node and potentially modify the chain. However, in a private, permissioned network, these mechanisms can be replaced with a simple proof of authority mechanism [5]. PoA is a modified version of PoS with identity as the only 'stake' [14]. We tested our smart contracts on a private test network using proof of authority.

\section{Ethereum blockchain and smart contracts}

A broad view of Ethereum might divide the world into three parts: blockchain, network trie roots, and trie data structures [Fig. 1b]. The blockchain logs the network's state at specific times, after transactions have altered the 


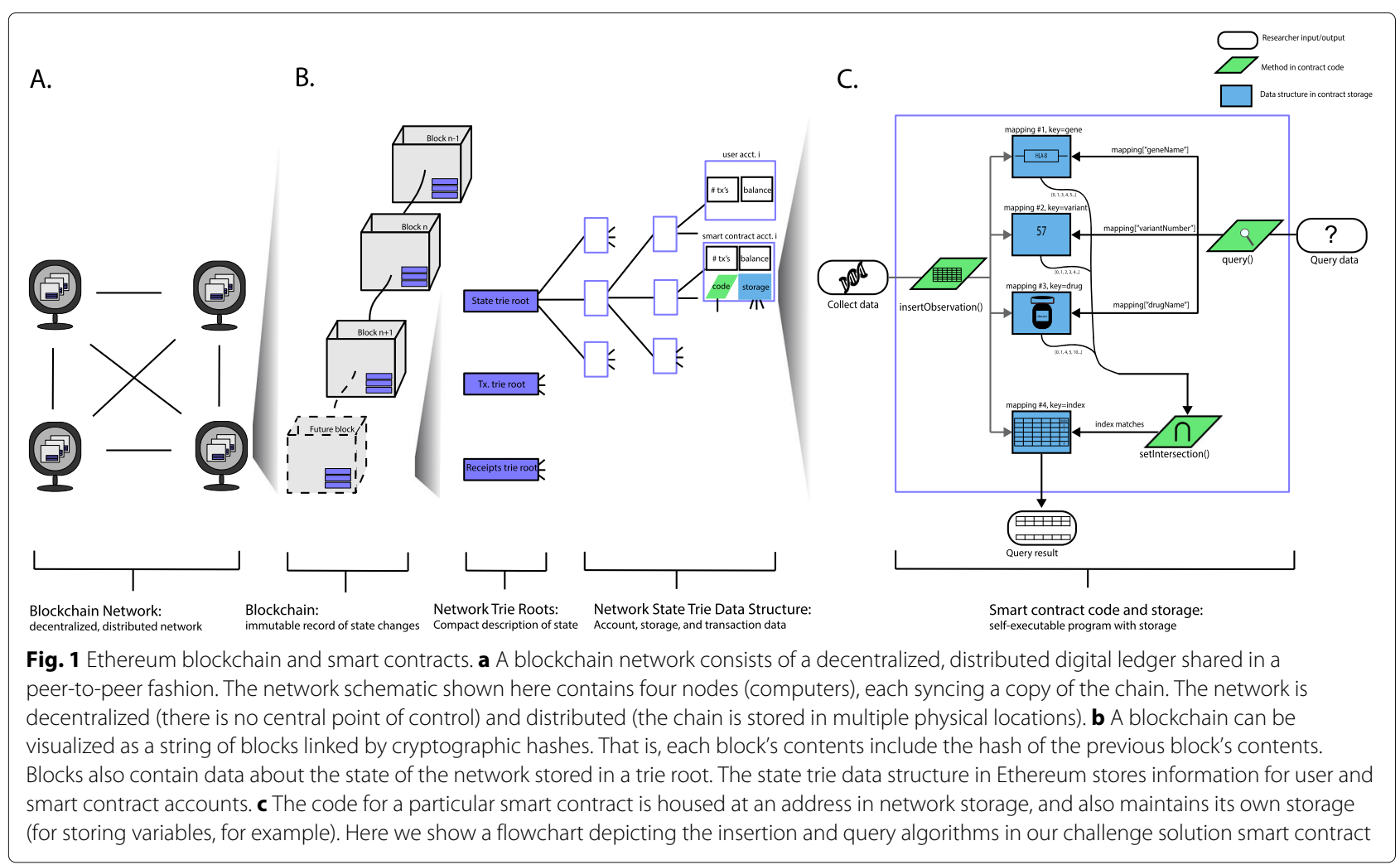

state. The state of the network is stored in merkle patricia trees each of which possess a top hash. Blocks in the chain store these top hash values, but do not store all the data in the blockchain network (it would be far too large). The state data is stored in a database layer using leveldb $[15,16]$. User account data and smart contract data (including the code and the actual data inserted via the code) are stored in these trie data structures, which are synced by "full" and "archival" nodes only (nodes which require significantly more computational power and storage) [16]. These nodes are integral to the health of the network. However, the Ethereum protocol also contains a "light" node option, in which only the block headers are synced [9].

Ethereum can handle a wide range of transactions via smart contracts, self-executable turing-complete programs which run in the Ethereum Virtual Machine (EVM) and maintain state in their own storage [8]. The EVM has a stack-based architecture, and can either store things on the stack (e.g. bytecode operations), in memory (e.g. temporary variables within functions), or in storage (e.g. permanent variables holding database entries). Each smart contract can read and write to its own storage only. In order to discourage developers from writing inefficient or unwieldy smart contracts, there is a 'gas' cost associated with each storage and retrieval command. Just as blockchain users have an address, a smart contract's state resides at a particular unique address in the global state of the Ethereum network, which users can call. If a user does not have enough gas, the contract call and corresponding transaction cannot be completed. Smart contracts provide an opportunity to develop applications with complex functionality in a blockchain network. We leveraged the flexibility of smart contract programming to create a challenge solution and alternate fastQuery solution that insert pharmacogenomics data in a custom way in contract storage to maximize storage and query efficiency.

\section{Network configuration}

We developed and tested our solutions in Truffle v5.1.18, a command line tool for Ethereum, which provided us with a built-in JavaScript test environment. Within this network, we tested insertion and querying with up to 10,000 entries in the database. Our setup constituted a "development network", which was separate from the public Ethereum network. This private configuration allowed us to develop and test our contracts extensively, without having to deploy a new contract each time. While network configuration can drastically affect performance in a Proof-of-Work scheme, it has minimal effects in a Proof-of-Authority scheme. Schaffer et al. demonstrate that increasing the number of nodes in a private, Proof-ofAuthority Ethereum network does not significantly affect performance [17]. 


\section{Chain initialization}

Chain initialization in Truffle is automated, and only requires setting basic parameters such as gas limit and network name in a config file. We used the default gas limit and price values for the development network (4712388 and 100 gwei, respectively). Together, gas limit and price values determine the maximum amount of Ether one can spend on transaction costs (one can spend no more than gas price * gas limit). In a proof of work scheme, the gas price also affects transaction speed, as miners will mine transactions with a more profitable gas price [18]. See [8] for a detailed explanation of the gas price and gas limit parameters.

\section{Challenge solution: an index-based, multi-mapping smart contract}

Our challenge solution utilizes four storage mappings, linked to one another by a unique integer ID assigned to each inserted observation in the database [Fig. 1c]. Mappings are similar to hash tables, and allow efficient key-value lookup. In the first of the four mappings, which we call the database, we store the pharmacogenomics data and assign to each entry a unique ID to serve as the mapping key. We store each observation in its own struct, a composite data type which can hold multiple fields. In the three other mappings, we use the gene names, variant numbers, and drug names as keys to an array of relevant IDs. Thus, given a gene name key, one can retrieve a list of IDs that can key into the database mapping and return an observation matching that particular gene name. We chose this implementation in order to reduce the number of loops required to check the data in the database and return matches, and thereby achieve time/memory efficient querying.

Challenge solution: insertion Data can be inserted into a smart contract via one-line commands in a JavaScript console or from an external script. We wrote an insertion function within the contract to insert a single observation for a given gene name-variant number-drug name combination. In our case the observation consists of the gene name, variant number, drug name, outcome (improved, unchanged, or deteriorated), suspected gene-outcome relation (true/false), and serious side effect (true/false). Upon passing in the observation, the function executes the following steps, (1) Convert each field of the observation to the desired data type for storage (e.g. it is more efficient to store strings as bytes32 types in storage); (2) if the gene name, variant, drug combination does not already exist in the database, add it to an array holding only the unique gene name, variant, and drug name combinations; (3) Use the gene name, variant number, and drug name to key into their respective mapping and append to the value array the counter as the ID, where counter is a globally updated variable; (4) push to the database mapping a struct with key-value pair: counter-[struct holding the observation]; (5) increment counter variable by one (see Algorithm 1).

We store the gene name, variant number, and outcome fields as bytes 32 variables, a fixed-size bytes array that uses less 'gas' in Ethereum relative to strings and is compatible with basic utilities in Solidity (for example, it is simpler to check the length of a bytes variable than a string variable using Solidity). We store the drug name as a string because many drug names are lengthy and can exceed the 32-character limit of the bytes32 data type. Booleans are straightforward in Solidity, and addresses are types to conveniently store the user or contract addresses on the blockchain.

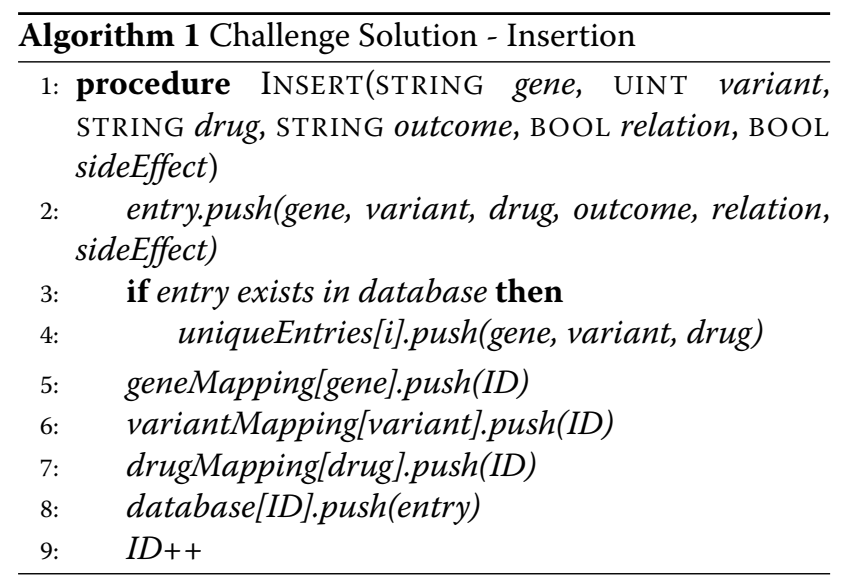

Challenge solution: query algorithm Our challenge solution can handle both three-field queries, where the fields are gene name, variant number, drug name, and wildcard queries. One can query by any combination of these three fields, or simply specify gene $=" * *$, variant $=$ "*", drug $=" * "$, which should return the entire contents of the database. To query, we first check how many fields have been queried, and for those that were, we use the query fields to key into the appropriate mapping and return the value associated with that key- an array of integer IDs corresponding to the ID of the data in the database mapping. If all fields were "*" we use all IDs currently in use to return the data from the database mapping. Otherwise, we determine which of the ID-arrays has the minimum length, and loop through it (since its contents will limit the results of the set intersection). For each entry in this minimum-length ID array, for every other field that was searched we loop through that ID array and check whether it matches the ID in the outer loop. At the end of the outer loop, if the number of matches is equal to the number of 
fields searched, then this integer is used to key into the database mapping and grab the struct value, which is then saved to a memory array. We then loop through the array of unique combinations and for each one pool the structs in the search results that match that unique combination. This allows us to output data in a useful way: for a given gene name, variant number, and drug name, how many observations are there, what number and percentage of cases saw serious side effects, what number and percentage suspected an interaction between the gene and drug, and what number and percentage saw an improved, deteriorated or unchanged outcome when administered the drug (see Algorithm 2).

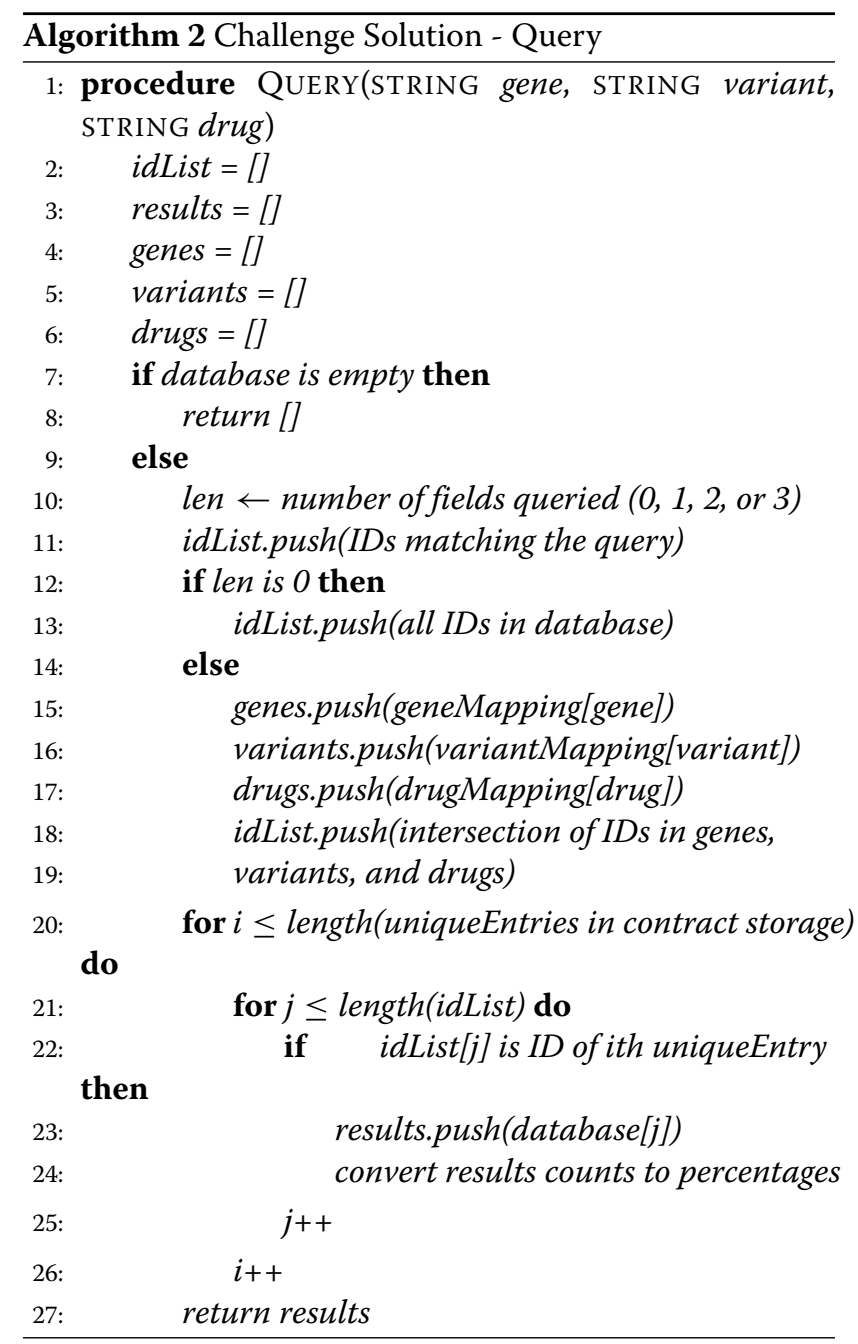

\section{fastQuery solution: a pooled, index-based, multi-mapping} smart contract

Rather than storing each observation in its own struct, our alternate, fastQuery solution stores a single struct for each unique gene-variant-drug relation. We utilize four storage mappings linked to one another by an ID assigned to each inserted observation in the database, and an additional fifth mapping for linking IDs to their unique gene-variantdrug relation. This alternate design reduces the number of IDed entries in the database, and prevents indefinite database growth (there is an infinite number of raw observations that can be inserted into our challenge solution, but a finite number of unique gene-variant-drug relations that exist). We chose this implementation in order to reduce the length of loops required to check the data in the database and return matches, and thereby further improve the query time.

fastQuery solution: insertion We wrote a new insertion function to insert a single observation for a given gene name-variant number-drug name combination. Upon passing in the observation, the function executes the following steps, (1) Convert each field of the observation to the desired data type for storage; (2) Use the input gene, variant, and drug as a combination key into the fifth mapping, which returns the ID for that unique relation; (3) Use the ID as a key into the database mapping and check whether this relation already exists in the database; (4) if not, fill in the name information from the input data, add the gene, variant, and drug names as keys in their respective mappings with the ID as the value, and increment the global ID counter by 1 ; (5) increment the total count, improved count, deteriorated count, suspected relation count, and side effect count fields of the relation struct in the database based on the inserted data. We store the variables as the same data types used in our challenge solution (see Algorithm 3).

fastQuery solution: query algorithm Our fastQuery solution can handle both unique and wildcard queries, like the challenge solution. Yet, with fastQuery, we do not need

\begin{tabular}{cc}
\hline Algorithm 3 fastQuery Solution - Insertion \\
\hline 1: & procedure INSERT(STRING gene, UINT variant, \\
& STRING drug, STRING outcome, BOOL relation, BOOL \\
& sideEffect) \\
2: & entryIdentity.push(gene, variant, drug) \\
3: & entryData.push(outcome, relation, sideEffect) \\
4: & ID $\leftarrow$ idKeeper[gene][variant][drug] \\
5: & if database[ID] exists then \\
6: & idKeeper[gene][variant][drug] $\leftarrow$ counter \\
7: & ID $\leftarrow$ counter \\
8: & database[ID].push(entryIdentity) \\
9: & genes[gene] $\leftarrow$ counter \\
10: & variants[variant] $\leftarrow$ counter \\
11: & drugs[drug] $\leftarrow$ counter \\
12: & counter ++ \\
13: & update database[ID] counts based on entryData \\
\hline
\end{tabular}


to iterate through an array of unique gene-variant-drug combinations because the data are already stored pooled in these unique relations. Thus, we simply obtain the matches using the challenge solution, convert the count data to percentages, and output the search result (see Algorithm 4 and Additional file 1).

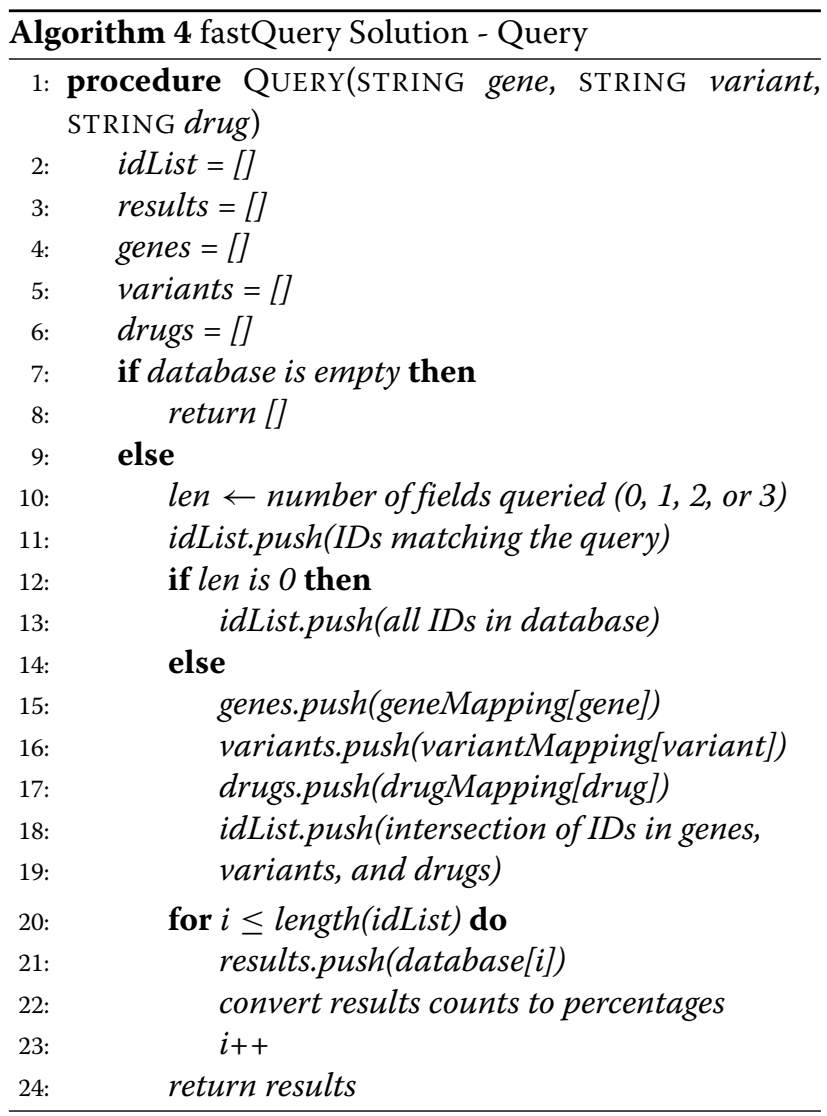

\section{Results}

We present two proof-of-concept solutions for storing pharmacogenomics data observations: our challenge solution, which we tested on databases of up to 1,000 entries, and an alternate fastQuery solution with improved performance, which we tested on databases of up to 10,000 entries. Both solutions were measured for its accuracy, time, space, and gas efficiency, and scalability [Figs. 2 and 3].

\section{Accuracy}

The Truffle environment allows testing from custom JavaScript scripts. Using assertions in JavaScript, we checked that the query results matched the fields queried for 100 random queries with zero, one, and two "AND"s. the challenge and fastQuery solutions.

\section{Time and space efficiency and scalability Insertion}

We measured the time, memory, and disk usage required to insert increasing amounts of data into contract storage, where each insertion is a single empirical observation of a gene-variant-drug interaction ( for example, gene $="$ CYP3A $5 "$, variant $=52$, drug $=$ pegloticase, outcome $=$ UNCHANGED, suspected gene-drugrelation $=$ true, serious side-effect $=$ true) $[$ Fig. 2]. We measured insertion time when inserting 200 entries at a time (with $1 \mathrm{~s}$ pauses between batches, subtracted from the reported times). We found that our challenge solution takes approximately $400 \mathrm{~ms}$ to insert 1,000 observations, with a linear time complexity. We found that the memory requirement for inserting 1,000 entries is $500 \mathrm{MB}$ per insertion. We found that disk space usage increases linearly with database entries, with approximately $0.92 \mathrm{MB}$ required to store 1,000 entries.

The fastQuery solution takes approximately $152 \mathrm{~ms}$ to insert 1,000 observations, with a linear time complexity [Fig. 2]. The memory requirement for inserting 1,000 entries into the chain is around $500 \mathrm{MB}$ per insertion. Disk space usage increases linearly with database entries, with approximately $1.6 \mathrm{MB}$ required to store 1,000 entries. While the challenge solution could only handle queries for less than 2,000 inserted entries, we were able to test performance of the fastQuery solution for databases up to 10,000 inserted entries in size.

\section{Querying}

We measured the performance of our query algorithm by testing the time, gas, and memory required to do a threefield query in a database with an increasing number of entries for 100 random queries [Fig. 3]. We found that our challenge solution takes an average of approximately $300 \mathrm{~ms}$ to complete a two-AND query from a database of 1,000 entries with an estimated linear time complexity. We also measured the memory requirement for a twoAND query from a database of 100 entries, and found that it was approximately $0.003 \mathrm{MB}$ per query and linear with increasing database entries. However, the challenge solution was not able to perform queries in our network configuration, on databases larger than 2,000 entries; and we were not able to measure gas or memory usage for queries on databases larger than 500 entries, due to the failure of the Truffle gas measurement function.

Our fastQuery algorithm showed improved query time [Fig. 3]. We found that it takes approximately $170 \mathrm{~ms}$ to complete a two-AND query from a database of 1,000 entries, and linear time with increasing number of database entries. For query memory requirement, it showed approximately $0.005 \mathrm{MB}$ per query from a database of 1,000 entries, and increased linearly with increasing database entries. Importantly, fastQuery 


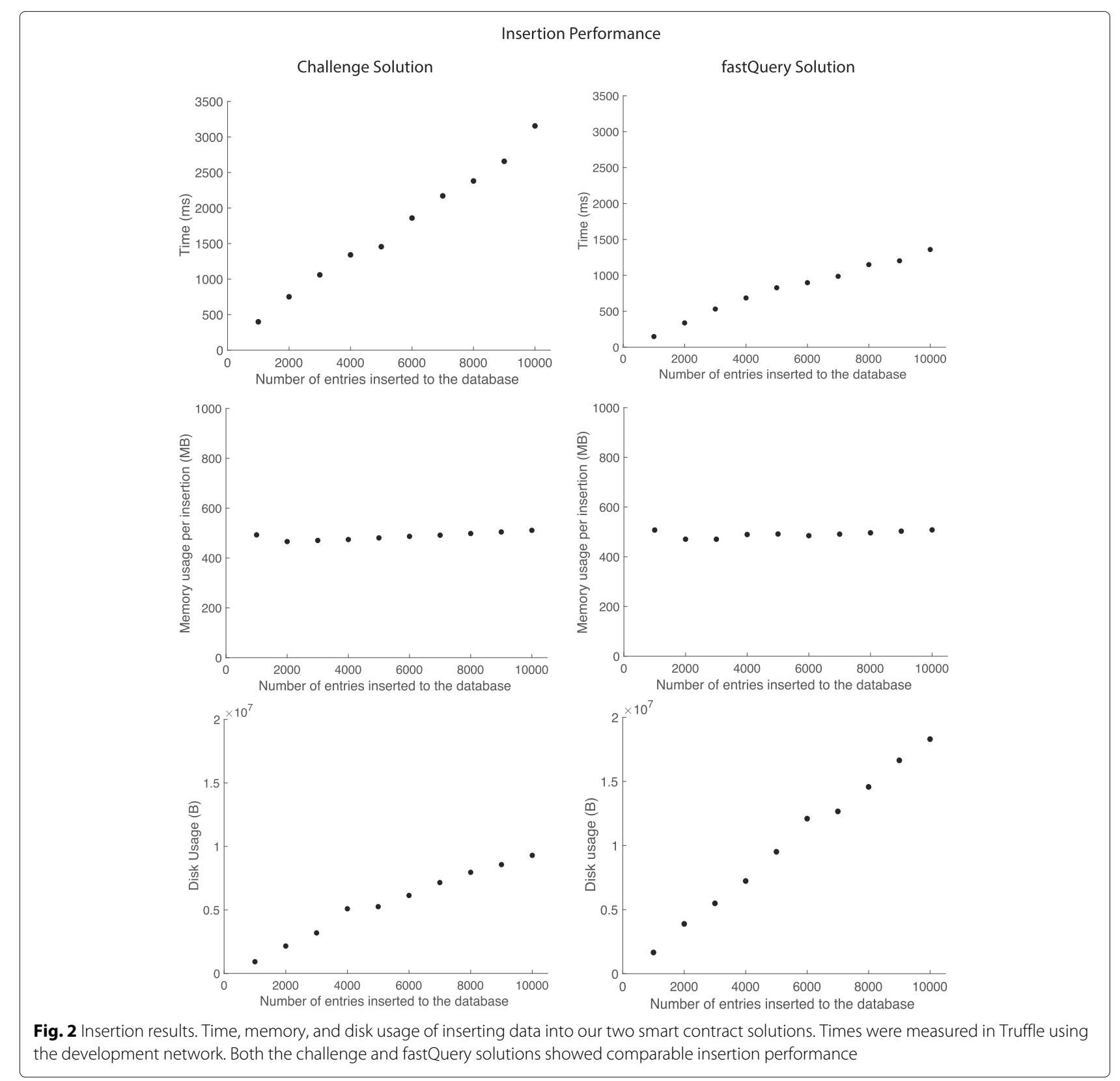

showed improved scalability. We were able to measure performance of fastQuery for databases of up to 10,000 entries. We found that it takes approximately $790 \mathrm{~ms}$ to complete a two-AND query from a database of 10,000 entries, and 0.01 MB.

\section{Effect of varying "ANDs" in query}

We investigated whether the time and memory efficiency of our two solutions varied with the number of "AND"s in a query [Fig. 3]. We checked whether for a database of 1,000 entries, changing the number of fields queried affects the time and memory requirement. We found that for one- and two-AND queries in the challenge solution, it takes an average of approximately $250 \mathrm{~ms}$ to query from a 1,000-entry database, while a 0-AND query takes an average of approximately $585 \mathrm{~ms}$ to do the same. We found memory usage per query to be comparable, regardless of the number of ANDs in the query.

For the fastQuery solution, we found that for one- and two-AND queries take an average of approximately 165 ms to query from a 1,000-entry database, while a 0-AND query takes an average of approximately $570 \mathrm{~ms}$ to do the same [Fig. 3]. Memory was approximately $0.005 \mathrm{MB}$ per query for a 2-AND query, $0.006 \mathrm{MB}$ for a 1 -AND query, and $0.01 \mathrm{MB}$ for a 0 -AND query in a database of 1,000 entries. One- and two-AND queries take an 


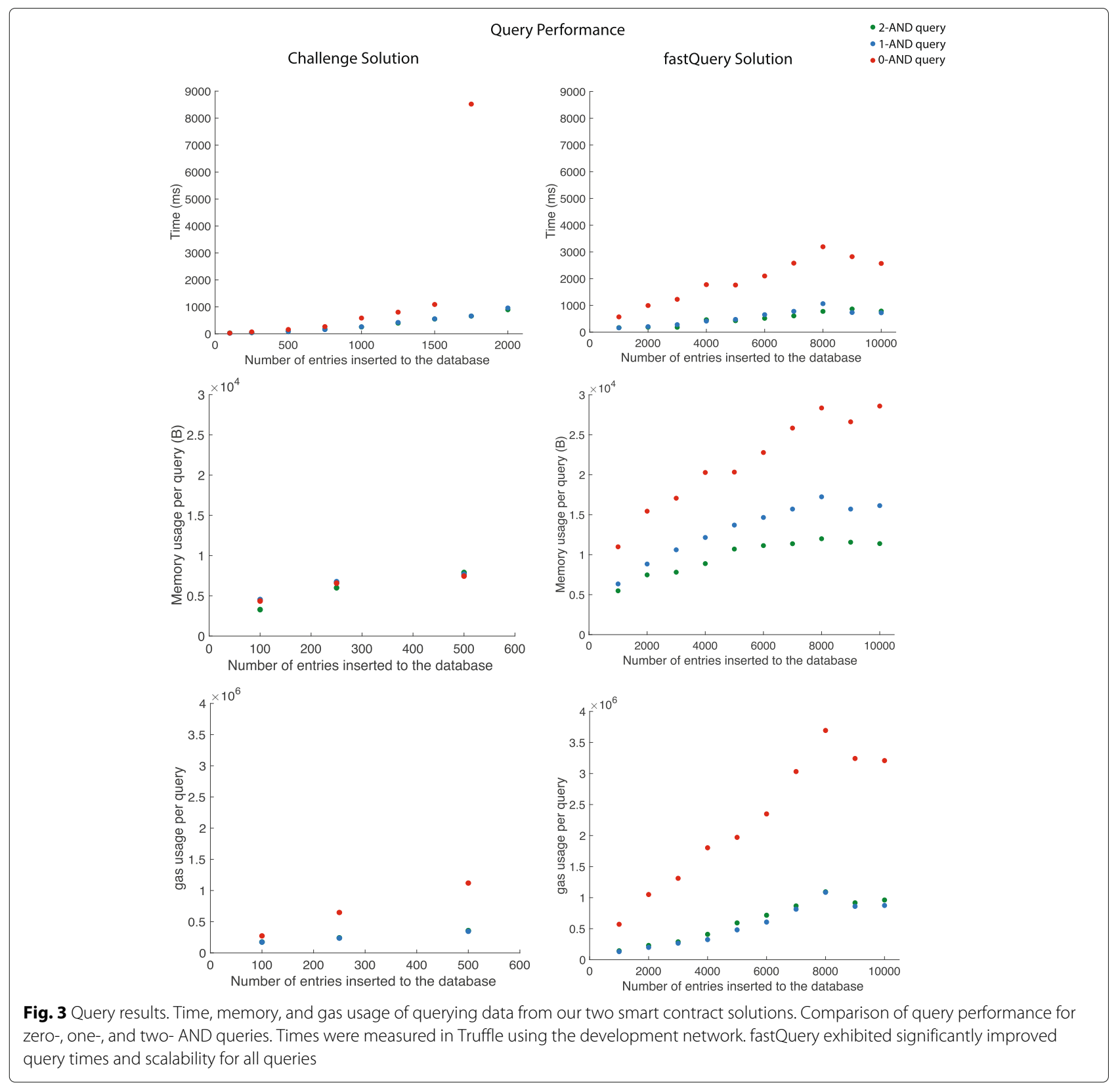

average of approximately $750 \mathrm{~ms}$ to query from a 10,000entry database, while a 0-AND query takes an average of approximately $2.5 \mathrm{~s}$ to do the same. Memory was approximately $0.011 \mathrm{MB}$ per query for a 2-AND query, $0.016 \mathrm{MB}$ for a 1-AND query, and $0.028 \mathrm{MB}$ for a 0 -AND query in a database of 10,000 entries.

\section{Discussion}

High-integrity, secure data maintenance is a major concern in biomedical research. In the case of pharmacogenomics, the data collected from clinical trials directly impacts medical treatment decisions. The integrity and security of the data is therefore critical, as loss or corruption will lead to misguided medical care. The 2019 IDASH Secure Genome Analysis competition proposed using smart contracts in a private Ethereum blockchain [19]. Such solutions would protect the data from loss in a single point of failure scenario and from accidental or intentional corruption. It also has broader implications, showing the potential for applying blockchain technology to solve real-world problems beyond cryptocurrency.

In this study, we presented two proof-of-concept solution to store pharmacogenomics data using the Ethereum blockchain platform. Both solutions addresses the need for security and accessibility in sharing these data, but also the practical need for time and memory efficiency 
for use in the real world. We showed that blockchain technology can not only offer security and immutability, but also efficiency and practicality. Although we were able to develop two efficient solutions as Ethereum smart contracts, development in Ethereum is far from easy. Setting up a private blockchain in Ethereum requires expert knowledge in the platform, and deploying the contract is a complex process. This process can be condensed into an external script, which reduces the need for expertise to use the platform. However there are still issues with bugs in Ethereum software such as in web3.js, the JavaScript API for Ethereum. We were able to overcome these software issues, but acknowledge the need for more stability in this platform before researchers begin using it for a shared database.

\section{Conclusion}

In summary, we presented a challenge solution for storing and querying pharmacogenomics data on the Ethereum blockchain in a smart contract and an alternate fastQuery solution with significantly improved query time and scalability. Our challenge solution made use of multiple mappings linked by unique integer identifiers. This design was advantageous, as it allowed querying by direct access to mappings (essentially hash tables), rather than by iterating through the entire database. Our fastQuery solution introduced pooled data storage, which further reduced query time by eliminating the need to check for unique gene-variant-drug combinations in the database when querying. Both solutions work well with scalable time and memory requirements up to 1,000 queries. However, although our challenge solution successfully stored the data, it required high amounts of gas in order to perform queries in a chain with more than 1,000 entries. Our fastQuery solution was successful up to 10,000 entries with scalable time, memory and gas requirements. Our algorithms had to be designed with Solidity's constraints in mind, such as the number of local variables permitted in a function, the 'gas' limit, and other peculiarities to Ethereum development. Our solutions demonstrate the potential for blockchain technology in the medical research community, but could be applied to a variety of other store and query problems.

\section{Supplementary information}

Supplementary information accompanies this paper at https://doi.org/10.1186/s12920-020-00732-x.

Additional file 1: Supplementary Information. A pdf file including a flowchart showing the design of the fastQuery smart contract

\section{Acknowledgements}

We thank iDASH organizers for providing an avenue to develop secure genome analysis applications.

\section{Authors' contributions}

G.G. and M.G designed the project and led the overall analysis. G.G and C.M.B designed the solutions and wrote the code. G.G. C.M.B and M.G. analyzed the results. G.G, C.M.B and M.G. wrote the manuscript. All authors read and approved of the final manuscript.

\section{Funding}

This work is supported by US National Institutes of Health U01 EB023686 grant and by the AL Williams Professorship Funds. Publication costs were funded by US National Institutes of Health U01 EB023686 grant.

\section{Availability of data and materials}

Source codes for insertion and querying, example input file, queries used in this study and the results for insertion and querying can be accessed at https://github.com/gersteinlab/iDASH19bc under GPL v3.0 license.

\section{Ethics approval and consent to participate}

Not Applicable.

\section{Consent for publication}

Not applicable.

\section{Competing interests}

The authors declare that they have no competing interests.

\section{Author details}

${ }^{1}$ Program in Computational Biology and Bioinformatics, Yale University, Whitney Avenue, 06520 New Haven, CT, USA. ${ }^{2}$ Department of Molecular Biophysics and Biochemistry, Yale University, Whitney Avenue, 06520 New Haven, CT, USA. ${ }^{3}$ Department of Computer Science, Yale University, Prospect Street, 06520 New Haven, CT, USA.

Received: 26 November 2019 Accepted: 25 May 2020

Published online: 01 June 2020

\section{References}

1. Mayo Clinic Center for Individualized Medicine. Pharmacogenomics: Drug-Gene Alerts. https://www.mayo.edu/research/centers-programs/ center-individualized-medicine/patient-care/pharmacogenomics/druggene-alerts. Accessed 28 May 2020.

2. Erlich $Y$, Narayanan A. Routes for breaching and protecting genetic privacy. Nat Rev Genet. 2014;15:409-21.

3. Kharpal A. Blockchain is 'one of the most overhyped technologies ever,' Nouriel Roubini says. 2018. https://www.cnbc.com/2018/03/06/ blockchain-nouriel-roubini-one-of-the-most-overhyped-technologiesever.html. Accessed 28 May 2020.

4. Kuo T, Kim H, Ohno-Machado L. Blockchain distributed ledger technologies for biomedical and health care applications. JAMIA. 2017;24(6):1211-20.

5. Nakamoto S. Bitcoin: A Peer-to-Peer Electronic Cash System. 2008. bitcoin.org/bitcoin.pdf. Accessed 28 May 2020.

6. Browne R. IBM partners with Nestle, Unilever and other food giants to trace food contamination with blockchain. 2017. https://www.cnbc.com/ 2017/08/22/ibm-nestle-unilever-walmart-blockchain-foodcontamination.html. Accessed 28 May 2020.

7. ConsenSys Ujo and Capitol Records bring blockchain innovation to music. 2018. https://media.consensys.net/consensys-ujo-and-capitolrecords-bring-blockchain-innovation-to-music-319f2c649790. Accessed 28 May 2020.

8. Wood G. Ethereum: a secure decentralised generalised transaction ledger byzantium version. 2019. https://ethereum.github.io/yellowpaper/paper. pdf. Accessed 28 May 2020.

9. A next-generation smart contract and decentralized application platform. 2019. https://github.com/ethereum/wiki/wiki/White-Paper. Accessed 28 May 2020.

10. Solidity. 2019. https://solidity.readthedocs.io/en/v0.5.12/. Accessed 28 May 2020.

11. Tso R, Liu Z, Hsiao J. Distributed E-Voting and E-Bidding Systems Based on Smart Contract. Electronics. 2019;8:422. https://doi.org/10.3390.

12. Zochowski M. Why proof-of-work is not viable in the long-term. 2019. https://medium.com/logos-network/why-proof-of-work-is-not-viablein-the-long-term-dd96d2775e99. Accessed 28 May 2020. 
13. King S, Nadal S. PPCoin: Peer-to-peer crypto-currency with proof-of-stake. 2012. https://decred.org/research/king2012.pdf. Accessed 28 May 2020.

14. POA Network Proof of authority: consensus model with identity at stake. 2017. https://medium.com/poa-network/proof-of-authority-consensusmodel-with-identity-at-stake-d5bd15463256. Accessed 28 May 2020.

15. McCallum T. Diving into Ethereum's world state. 2018. https://medium. com/cybermiles/diving-into-ethereums-world-state-c893102030ed. Accessed 28 May 2020.

16. The Ethereum world - how its data is stored. 2018. https://github.com/ tpmccallum/ethereum_database_research_and_testing/blob/master/ leveldb/leveldb.md. Accessed 28 May 2020.

17. Performance and Scalability of Private Ethereum Blockchains. 2019 https://publik.tuwien.ac.at/files/publik_280601.pdf. Accessed 28 May 2020.

18. web3j - Transactions. 2020. https://web3j.readthedocs.io/en/latest/ transactions.html. Accessed 28 May 2020.

19. IDASH Privacy and Security Workshop 2019 - secure genome analysis competition. 2019. http://www.humangenomeprivacy.org/2019/ competition-tasks.html. Accessed 28 May 2020.

\section{Publisher's Note}

Springer Nature remains neutral with regard to jurisdictional claims in published maps and institutional affiliations.

Ready to submit your research? Choose BMC and benefit from:

- fast, convenient online submission

- thorough peer review by experienced researchers in your field

- rapid publication on acceptance

- support for research data, including large and complex data types

- gold Open Access which fosters wider collaboration and increased citations

- maximum visibility for your research: over $100 \mathrm{M}$ website views per year

At BMC, research is always in progress.

Learn more biomedcentral.com/submissions 